University of Warwick institutional repository: http://go.warwick.ac.uk/wrap This paper is made available online in accordance with publisher policies. Please scroll down to view the document itself. Please refer to the repository record for this item and our policy information available from the repository home page for further information.

To see the final version of this paper please visit the publisher's website. Access to the published version may require a subscription.

Author(s): C. J. Emeleus, M. A. Sadeghzadeh, P. J. Phillips, E. H. C. Parker, T. E. Whall, M. Pepper, and A. G. R. Evans

Article Title: Back gating of a two-dimensional hole gas in a SiGe quantum well

Year of publication: 1997

Link to published version: http://dx.doi.org/10.1063/1.118729

Publisher statement: none 


\title{
Back gating of a two-dimensional hole gas in a SiGe quantum well
}

\author{
C. J. Emeleus, ${ }^{\text {a) }}$ M. A. Sadeghzadeh, P. J. Phillips, E. H. C. Parker, and T. E. Whall \\ Department of Physics, University of Warwick, Coventry CV4 7AL, United Kingdom \\ M. Pepper \\ Cavendish Laboratory, Cambridge CB3 OHE, United Kingdom
}

A. G. R. Evans

Department of Electronics and Computer Science, University of Southampton, Southampton SO17 1BJ, United Kingdom

(Received 20 November 1996; accepted for publication 4 February 1997)

\begin{abstract}
A device comprising a low-resistivity, n-type, Si substrate as a back gate to a p-type (boron), remote-doped, SiGe quantum well has been fabricated and characterized. Reverse and forward voltage biasing of the gate with respect to the two-dimensional hole gas in the quantum well allows the density of holes to be varied from $8 \times 10^{11} \mathrm{~cm}^{-2}$ down to a measurement-limited value of $4 \times 10^{11} \mathrm{~cm}^{-2}$. This device is used to demonstrate the evolution with decreasing carrier density of a re-entrant insulator state between the integer quantum Hall effect states with filling factors 1 and 3. (C) 1997 American Institute of Physics. [S0003-6951(97)03514-6]
\end{abstract}

The introduction of back-gating techniques in semiconductor device fabrication has extended significantly the range of physical phenomena it is possible to investigate in lowdimensional electronic systems. By placing the means of varying the carrier density of a two-dimensional electron gas (2DEG) away from its conventional location on the surface of the heterostructure (either a metal gate in direct contact with the semiconductor, or with an insulating layer between the two) the latter area is left free for patterning. Some important examples of the applications of back gating are: (i) the study of electron transport through one-dimensional (1D), quantum-size constrictions, which are themselves defined using pairs of surface gates to selectively deplete areas of 2DEG, with independent control of the 1D gas density maintained via the back-gate voltage; ${ }^{1}$ and (ii) the use of complementary front and back gates to switch on and off parallel, closely spaced 2DEGs, which is the basis of a method of making independent contact to only one of the two gases, ${ }^{2}$ opening up many possibilities for the study of in-plane and tunnelling transport phenomena in coupled 2DEG systems. ${ }^{3}$

The first attempt to back gate a 2DEG involved fixing a metal plate to the bottom of a modulation-doped GaAs/ $\mathrm{Al}_{x} \mathrm{Ga}_{1-x} \mathrm{As}$ heterostructure containing the 2DEG. ${ }^{4}$ The disadvantage with this approach was that the inherently large distance between the gate and 2DEG (in this case, a substrate thickness of $0.4 \mathrm{~mm}$ ) necessitated application of large gatechannel bias voltages $\left(\sim 10^{2}-10^{3} \mathrm{~V}\right)$ to obtain significant modulation of the 2D electron density. Subsequently, devices were fabricated in which the back gate was either a low resistivity substrate ${ }^{5,6}$ and/or a highly doped layer as the first stage of epitaxy, $5,7,8$ thereby bringing the gate within $1 \mu \mathrm{m}$ or so of the conducting channel and increasing by orders of magnitude the sensitivity of the 2DEG density to change in the gate-channel bias voltage. There are two reports in the literature of back gating of $\mathrm{Si} / \mathrm{SiGe}$ heterostructures, ${ }^{9,10}$ both

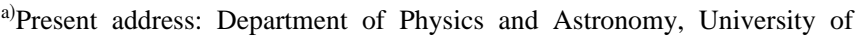
Glasgow, Glasgow G12 8QQ, United Kingdom. Electronic mail: emeleus@physics.gla.ac.uk
}

of which involved evaporation of metal onto the backside of a low conductivity substrate with a high mobility Si-channel 2DEG structure on the front (in essence, the original approach to back-gating $2 \mathrm{DEGs}$ in III-V heterostructures ${ }^{4}$ ).

For the present study, we chose to grow a p-type modulation-doped $\mathrm{Si} / \mathrm{SiGe}$ heterostructure on a low-resistivity n-type Si substrate. Holes are constrained to move in a SiGe quantum well approximately triangular in shape, which is defined by (i) the offset in the valence band at the $\mathrm{Si} / \mathrm{SiGe}$ heterointerface nearest the B-doped capping layer, and (ii) the electric field established in the device due to the effects of hole transfer to the SiGe quantum well and also p-n depletion between the p-type dopant supply layer and the n-type substrate. A change of electric field in the well induced by biasing of the n-type gate (substrate) with respect to the p-type well, and thereby an adjustment to the quantum well shape together with a shift of the valence band edge in the well relative to the Fermi energy, leads to modulation of the two-dimensional hole gas (2DHG) density. For in-plane carrier-transport measurements the practical operating range of such a device, which we consider as a modified $p-i-n$ diode, lies between the forward bias turn-on and reverse-bias breakdown points, provided that leakage currents in this region are negligible compared with the desired source-drain current along the quantum well. To the best of our knowledge this specific method of gating a 2D gas has been reported only once before, as introduced by Churchill et al., ${ }^{8}$ who deposited a high conductivity Be-doped GaAs layer on a GaAs substrate as the first stage of epitaxy, using this as a back gate to a $2 \mathrm{DEG}$ located at an $\mathrm{Al}_{x} \mathrm{Ga}_{1-x} \mathrm{As} /$ GaAs heterojunction.

A cross-sectional view of the device fabricated in this work is shown in Fig. 1(a). The following layers were deposited by solid-source molecular beam epitaxy (MBE) on a 100-mm-diam, n-type (100) $\mathrm{Si}$ substrate of resistivity $<0.01 \Omega \mathrm{cm}$ : first of all, $430 \mathrm{~nm}$ of $\mathrm{Si}$; next, $40 \mathrm{~nm}$ of $\mathrm{Si}_{0.8} \mathrm{Ge}_{0.2}$; then a $10 \mathrm{~nm}$ spacer layer of $\mathrm{Si}$; and finally, a $\mathrm{Si}$ :B doping layer of thickness $50 \mathrm{~nm}$, with an acceptor concentration $N_{A}=2 \times 10^{18} \mathrm{~cm}^{-3}$.

The first step in device fabrication was to make electrical 
(a)

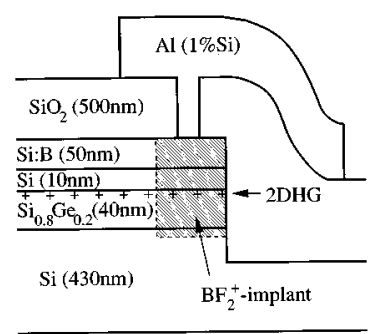

$\mathbf{n}^{+}-$Si substrate

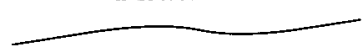

(b)

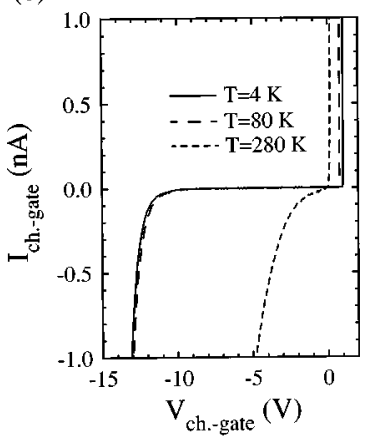

FIG. 1. (a) Cross section of part of a Hall bar device. (b) Current-voltage characteristics for the channel (2DHG)-gate(substrate) $p-i-n$ diode at various temperatures.

contact to the buried 2DHG. Implantation of $\mathrm{BF}_{2}^{+}$ions $(75$ $\mathrm{keV}$ energy, dosage of $1 \times 10^{15} \mathrm{~cm}^{-2}$ ) was chosen in preference to metal deposition plus an alloying anneal, in order to avoid the possibility of the contact spiking through to the substrate and thereby shorting the $2 \mathrm{DHG}$ to the gate. The implanted regions were recrystallized by an anneal in flowing $\mathrm{N}_{2}$ at $750{ }^{\circ} \mathrm{C}$ for $30 \mathrm{~min}$, a procedure which was known not to relax the strained $\mathrm{Si}_{0.8} \mathrm{Ge}_{0.2}$ layer. ${ }^{11}$ Hall bar shaped mesa areas of $2 \mathrm{DHG}$ were then defined using reactive-ion etching with $\mathrm{CF}_{4}$, to a depth of $300 \mathrm{~nm}$. The mask design ensured partial self-alignment of the implant with the mesa boundary, thereby preventing the occurrence of (unwanted) Corbino effects in the magneto-resistance under quantum Hall effect conditions, these being associated with complete loops of 2DHG around contacts. Finally, a passivating layer of $\mathrm{SiO}_{2}, 500 \mathrm{~nm}$ thick, was deposited on the wafer, windows opened in the oxide above the implants using hydrofluoric acid and $\mathrm{Al}: \mathrm{Si}(1 \%)$ evaporated onto the wafer and patterned into contact pads and tracks by etching with orthophosphoric acid.

Current-voltage $(I-V)$ characteristics for the gatechannel $(p-i-n)$ diode are shown in Fig. 1(b). At a temperature $T=4 \mathrm{~K}$, the abrupt turn-on voltage of $1.0 \mathrm{~V}$ in forwardbias and reverse breakdown threshold around $10 \mathrm{~V}$, with a leakage current of less than $10 \mathrm{pA}$ in the intermediate region, constitute very favorable conditions for in-plane transport experiments on the $2 \mathrm{DHG}$. These were carried out using an ac lock-in method at a frequency of $7 \mathrm{~Hz}$, with currents of 2 $\mathrm{nA}$ or less, in order to avoid electron heating effects at the lowest lattice temperatures. Two Hall bars were used, of dimensions $15 \mu \mathrm{m} \times 170 \mu \mathrm{m}$ and $30 \mu \mathrm{m} \times 280 \mu \mathrm{m}$, with corresponding distances between voltage probes used for $R_{x x}$ measurement of 85 and $90 \mu \mathrm{m}$. No discrepancy was seen between results from the two different geometry devices.

Magnetotransport measurements of $R_{x x}$ and $R_{x y}$ were made in the range $B=0-12 \mathrm{~T}$ at $T=0.35 \mathrm{~K}$. The dependence of $R_{x x}$ on $B$ for various gate voltages $\left(V_{g}\right)$ is shown in Fig. 2, with the shifts in positions of Shubnikov-de Haas oscillation minima reflecting the dependence of sheet density $\left(n_{s}\right)$ on $V_{g}$. Values of $n_{s}$ were determined from the data for forward- and small reverse-bias voltages in the usual way, by relating positions of the various $R_{x x}$ oscillation minima to filling factors $(\nu)$ using the defining relationship $\nu=n_{s} /$
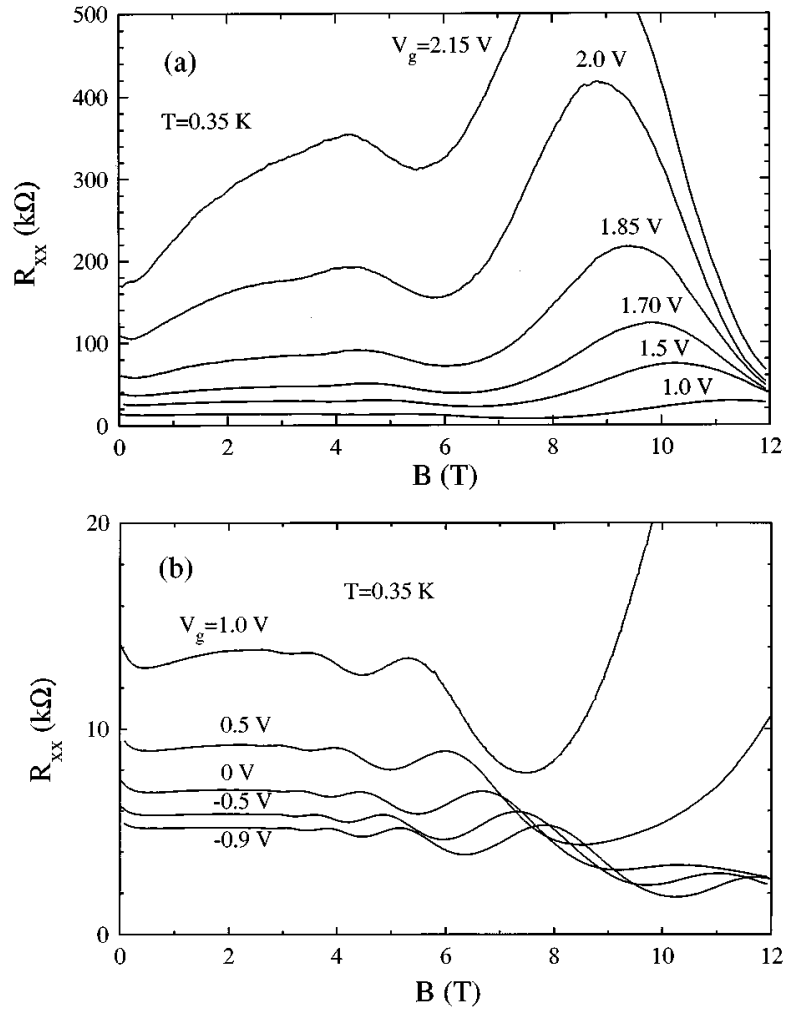

FIG. 2. Magnetoresistance oscillations in $R_{x x}$ as a function of gate voltage: (a) $V_{g}=1.0 \rightarrow 2.15 \mathrm{~V}$; (b) $V_{g}=-0.9 \rightarrow+1.0 \mathrm{~V}$.

$(e B / h)$, where $e$ is the electron charge and $h$ is Planck's constant. For $V_{g} \geqslant 1.5 \mathrm{~V}, n_{s}$ could only be estimated from the value of $B$ field at the single, observable $R_{x x}$ minimum (at $\nu=3)$. It was not possible to use the low $B$-field Hall voltage ( $V_{x y}$ ) to determine a value of $n_{s}$ since the low current used to avoid sample heating meant that $V_{x y}$ was not well resolved in our experiment. In any case, the Hall resistance $R_{x y}$ at small B fields is sensitive to quantum corrections due to disorder (i.e., enhanced interaction effects ${ }^{12}$ ), which must be extracted in order to obtain a meaningful estimation of $n_{s}$, while the analysis of high $B$-field $R_{x x}$ oscillations has no such complications. A plot of the dependence of $n_{s}$ on $V_{g}$ in Fig. 3 shows an approximately linear relationship, which is expected since the thickness of doped substrate depleted as $V_{g}$ is swept is a very small fraction of the gate-channel sepa-

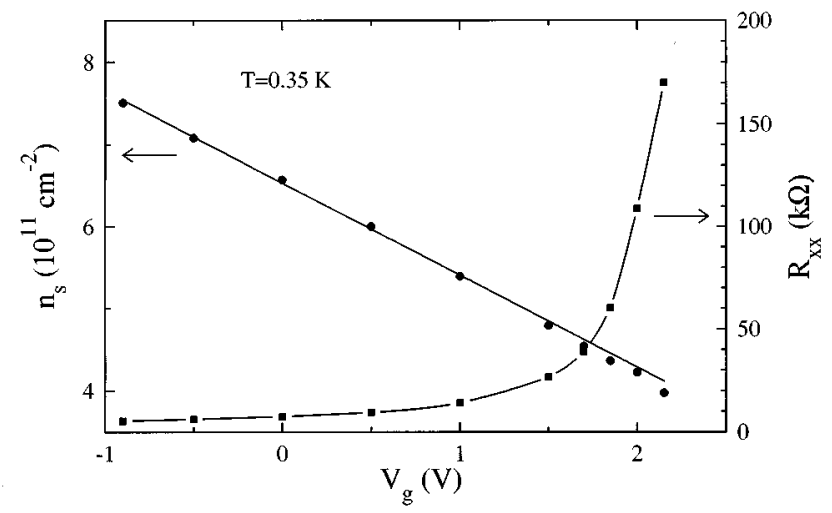

FIG. 3. Dependence of $n_{s}$ and $R_{x x}(B=0 \mathrm{~T})$ on gate voltage (data extracted from Fig. 2), plus a fit to the $n_{s}-V_{g}$ data (the line through the $R_{x x}$ data points is for guidance only). 


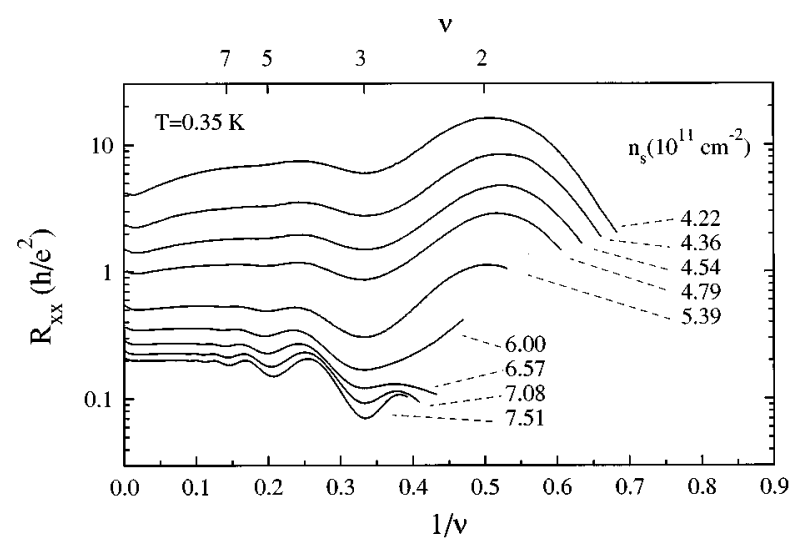

FIG. 4. Magnetoresistance data (units of $h / e^{2}=26 \mathrm{k} \Omega$ ) against reciprocal filling factor $1 / \nu$.

ration $(\leqslant 1 \%)$, such that the capacitance of this $p^{+}-i-n^{+}$ diode is effectively independent of $V_{g}$. In contrast with this, the variation of $R_{x x}$ with $V_{g}$ at $B=0 \mathrm{~T}$ is highly non-linear (Fig. 3): for $V_{g} \leqslant 1.5 \quad \mathrm{~V} \quad$ (corresponding to $n_{s} \geqslant 5 \times 10^{11} \mathrm{~cm}^{-2}$ and $R_{x x} \leqslant 25 \mathrm{k} \Omega$ ) $R_{x x}$ changes very slightly with $n_{s}$, but for higher $V_{g}$ there is a rapid increase in $R_{x x}$ with decreasing $n_{s}$. This transition, which is from weak to strong localisation of holes, occurs when $n_{s}$ (proportional to the Fermi energy of the 2DHG) falls below some threshold value corresponding to $R_{x x} \sim h / e^{2}(=26 \mathrm{k} \Omega$ ), and is familiar in other 2D systems. ${ }^{13}$

The dependence of $n_{s}$ on $V_{g}$ has been modelled by solving self-consistently a set of expressions based on the solutions of Poisson's and Schrödinger's equations applied to this heterostructure. The fit to the $n_{s}-V_{g}$ data in Fig. 3 is obtained firstly by choosing either a $23 \%$ Ge composition in the alloy (instead of the intended value of 20\%) or adjusting the Si spacer thickness between the dopant supply layer and SiGe from the $10 \mathrm{~nm}$ intended to $8 \mathrm{~nm}$ (both of these discrepancies are almost within the uncertainty expected for the growth). Second, however, it is necessary to set the thickness of Si between the SiGe layer and substrate to $525 \pm 30 \mathrm{~nm}$, which disagrees with the value of $430 \mathrm{~nm}$ expected from the growth. Secondary ion mass spectrometry yielded a value for this thickness of $485 \pm 20 \mathrm{~nm}$, which is in good agreement with our electrical analysis of the device operation.

We note that, although our measurements of $n_{s}$ are limited at higher reverse-bias voltages by very large sample resistances (a low carrier mobility, leading to loss of Shubnikov-de Haas oscillations in $R_{x x}$ ), an extrapolation of the $n_{s}-V_{g}$ data in Fig. 3 to $n_{s}=0$ together with the apparent reverse-bias breakdown voltage of $10 \mathrm{~V}$ [Fig. 1(b)] indicate that it should be possible to fully deplete the channel of holes without breakdown occurring. This means that the technique of $p-i-n$ biasing in a $\mathrm{Si} / \mathrm{SiGe}$ heterostructure may be used as a current switch, for example, such that the design and fabrication of more complex devices, similar to those mentioned at the beginning of this letter, may be undertaken.

Using the values of $n_{s}$ determined previously, the $R_{x x}-B$ data have been re-plotted as $R_{x x}-\nu$ in Fig. 4, which illustrates the appearance of a peak in $R_{x x}$ around $\nu=2$ for $n_{s} \leqslant 6 \times 10^{11} \mathrm{~cm}^{-2}$ and the gradual increase of its magnitude with decreasing $n_{s}$. This feature, caused by the formation of a re-entrant insulator state, has been observed previously in un-gated $\mathrm{Si} / \mathrm{SiGe}$ p-channel devices, ${ }^{14-16}$ and its origin explained on the basis of long range potential fluctuation in a sample. ${ }^{17}$ Experiments at higher magnetic fields are in progress, so that filling factor $\nu=1$ can be reached at all the values of $n_{s}$ accessible in the device (see Fig. 4), such that evolution of the insulating state around $\nu=2$ can be investigated in detail.

This work was funded by the United Kingdom Engineering and Physical Sciences Research Council (EPSRC). The authors would like to thank the staff at the EPSRC-funded Southampton University Microelectronics Centre, where the device fabrication took place.

${ }^{1}$ A. R. Hamilton, J. E. F. Frost, C. G. Smith, M. J. Kelly, E. H. Linfield, C. J. B. Ford, D. A. Ritchie, G. A. C. Jones, M. Pepper, D. G. Hasko, and H. Ahmed, Appl. Phys. Lett. 60, 2782 (1992).

${ }^{2}$ J. P. Eisenstein, L. N. Pfeiffer, and K. W. West, Appl. Phys. Lett. 57, 2324 (1990)

${ }^{3}$ See, e.g., the following: T. J. Gramila, J. P. Eisenstein, A. H. MacDonald, L. N. Pfeiffer, and K. W. West, Phys. Rev. Lett. 66, 1216 (1991); N. K. Patel, E. H. Linfield, K. M. Brown, M. P. Grimshaw, D. A. Ritchie, G. A. C. Jones, and M. Pepper, Appl. Phys. Lett. 64, 3018 (1994); and K. M. Brown, E. H. Linfield, D. A. Ritchie, G. A. C. Jones, M. P. Grimshaw, and M. Pepper, ibid. 64, 1827 (1994).

${ }^{4}$ H. L. Störmer, A. C. Gossard, and W. Wiegmann, Appl. Phys. Lett. 39, 493 (1981).

${ }^{5}$ U. Meirav, M. Heiblum, and F. Stern, Appl. Phys. Lett. 52, 1268 (1988).

${ }^{6}$ A. Kastalsky, F. Peeters, W. K. Chan, L. T. Florenz, and J. P. Harbison, Appl. Phys. Lett. 59, 1708 (1991).

${ }^{7}$ E. H. Linfield, G. A. C. Jones, D. A. Ritchie, and J. H. Thompson, Semicond. Sci. Technol. 8, 415 (1993).

${ }^{8}$ A. C. Churchill, M. P. Grimshaw, D. A. Ritchie, E. H. Linfield, and G. A. C. Jones, Semicond. Sci. Technol. 8, 1596 (1993).

${ }^{9}$ K. Ismail, M. Arafa, F. Stern, J. O. Chu, and B. S. Meyerson, Appl. Phys. Lett. 66, 842 (1995).

${ }^{10}$ A. Yutani and Y. Shiraki, Semicond. Sci. Technol. 11, 1009 (1996).

${ }^{11}$ R. Hull and J. C. Bean, Appl. Phys. Lett. 55, 1900 (1989).

${ }^{12}$ B. L. Altshuler, D. Khmel'nitzkii, A. I. Larkin, and P. A. Lee, Phys. Rev. B 22, 5142 (1980).

${ }^{13}$ e.g., the 2DEG inversion layer in a Si metal-oxide-semiconductor fieldeffect transistor (MOSFET): see M. J. Uren, R. A. Davies, M. Kaveh, and M. Pepper, J. Phys. C 14, 5737 (1981).

${ }^{14}$ S. I. Dorozhkin, C. J. Emeleus, T. E. Whall, and G. Landwehr, Phys. Rev. B 52, 11638 (1995).

${ }^{15}$ S. I. Dorozhkin, C. J. Emeleus, T. E. Whall, G. Landwehr, and O. A. Mironov, Pis'ma Zh. Éksp. Teor. Fiz. 62, 511 (1995) [JETP Lett. 62, 534 (1995)].

${ }^{16}$ F. F. Fang, R. B. Dunford, R. G. Clark, R. Newbury, R. P. Starrett, V. A. Stadnik, A. V. Skougarevsky, R. H. McKenzie, J. O. Chu, K. E. Ismail, and B. S. Meyerson, High Magnetic Fields in the Physics of Semiconductors, Proceedings of 11th International Conference (World Scientific, Singapore, 1995), pp. 620-623.

${ }^{17}$ S. I. Dorozhkin, Pis'ma Zh. Éksp. Teor. Fiz. 60, 578 (1994) [JETP Lett. 60, 595 (1994)]. 